Quentin Goossens ORCID iD: 0000-0002-3853-5495

Kathleen Denis ORCID iD: 0000-0002-6492-9607

\title{
ACOUSTIC ANALYSIS TO MONITOR IMPLANT SEATING AND EARLY DETECT FRACTURES IN CEMENTLESS THA: AN IN VIVO STUDY
}

\author{
Quentin Goossens ${ }^{1}$ Leonard Pastrav ${ }^{1}$ Jorg Roosen $^{2}$ Michiel Mulier $^{3}$ Wim Desmet $^{4}$ \\ Jos Vander Sloten ${ }^{1}$ Kathleen Denis ${ }^{1}$ \\ ${ }^{1}$ KU Leuven, Department of Mechanical Engineering, Biomechanics Section, Leuven, \\ Belgium \\ ${ }^{2}$ KU Leuven, Faculty of Medicine, Steps Skills Centre, Leuven, Belgium \\ ${ }^{3}$ University Hospital Leuven, Division of Orthopaedics, Leuven, Belgium \\ ${ }^{4}$ KU Leuven, Department of Mechanical Engineering, MSD Section, Leuven, Belgium \\ Corresponding author:
}

Quentin Goossens, e-mail: quentin.goossens@kuleuven.be, address: Andreas Vesaliusstraat 13 box 2600, 3000 Leuven, Belgium, tel.: +32 16301016 .

\section{Running title:}

ACOUSTIC ANALYSIS IN CEMENTLESS THA

\section{Author contributions statement:}

All authors have contributed substantially to the research design or the acquisition, analysis or interpretation of data and the drafting of the paper or revised it critically.

All authors have read and approved the final submitted manuscript.

This article has been accepted for publication and undergone full peer review but has not been through the copyediting, typesetting, pagination and proofreading process, which may lead to differences between this version and the Version of Record. Please cite this article as doi: $10.1002 /$ jor.24837. 


\begin{abstract}
The initial stability of cementless total hip arthroplasty (THA) implants is obtained by an interference fit that allows osseointegration for a long term secondary stability of the implant. Yet, finding the insertion endpoint that corresponds to an appropriate initial stability is currently often based on a number of subjective experiences of the orthopaedic surgeon, which can be challenging.

In order to assist the orthopaedic surgeons in their pursuit to find this optimal initial stability, this study aims to determine whether the analysis of sound that results from the implant insertion hammer blows can be used to objectively monitor the insertion process of cementless THA implants. An in vivo study was conducted.
\end{abstract}

The experimental results revealed vibro-acoustic behavior sensitive to implant seating, related to the low frequency content of the response spectra. This sensitive lowfrequency behavior was quantified by a set of specific vibro-acoustic features and metrics that reflected the power and similarity of the low-frequency response. These features and metrics allowed monitoring the implant seating and their convergence agreed well with the endpoint of insertion as determined by the orthopaedic surgeon. Intra-operative fractures caused an abrupt and opposite change of the vibro-acoustic behavior prior to the notification of the fracture by the orthopaedic surgeon. The observation of such an abrupt change in the vibro-acoustic behavior can be an important early warning for loss of implant stability.

The presented vibro-acoustic measurement method shows potential to serve as a decision supporting source of information as it showed to reflect the implant seating.

Keywords: Cementless Total Hip Arthroplasty, Primary implant stability, Vibroacoustic analysis

This article is protected by copyright. All rights reserved. 


\section{INTRODUCTION}

Cementless fixation is increasingly used as a fixation method in total hip arthroplasty (THA) ${ }^{1}$. Cementless implants obtain their intra-operative stability by an interference fit where sufficient bone-implant contact is an important factor to ensure the longterm outcome of the implant ${ }^{2}$. This interference fit is obtained by inserting the implant and a slightly undersized cavity by means of hammer blows. Finding the optimal endpoint of implant insertion is a key factor for a successful outcome of the surgery since it allows proper osseointegration of the implant ${ }^{3,4}$. The orthopaedic surgeons use different feedback mechanisms to find the optimal endpoint of implant insertion. Tactile and visual experiences are used, but also acoustic cues that result from hammering the implant in the bone are an important feedback mechanism for the surgeon. However, the interpretation of these different feedback mechanisms is of a subjective nature and may lead to uncertainties and differences between observers with a risk of improper primary implant fixation. Insufficient fixation may lead to early loosening of the implant while excessively press-fitting the implant in the bone may cause intra-operative fractures. Intra-operative fractures are reported to occur in 3 - $5 \%$ of primary THA and up to $20 \%$ in revision THA ${ }^{5-7}$. In order to support surgeons in their pursuit for optimal primary implant stability, the development of objective measurement methods to monitor the implant stability real-time has been focus of several studies. Vibration analysis has proven to be an effective approach in vitro ${ }^{8-11}$ and in vivo ${ }^{12}$. However, these approaches require contact-based sensors and actuators which have a number of disadvantages; an increased risk of infections, specific sensor (dis)mounting procedures with necessary modifications to the implant and corresponding time loss. In an attempt to overcome these challenges, contactless vibro-acoustic methods have been the subject of a limited number of studies ${ }^{13-21}$.

This article is protected by copyright. All rights reserved. 
Most of these studies demonstrated the potential of using acoustic methods but did not provide clearly defined protocols or criteria regarding the detection of the endpoint of insertion or regarding intra-operative fracture warning.

A prior in vitro study by the authors revealed sensitive vibro-acoustic behavior during femoral broach insertion ${ }^{22}$. As a next step, this study aims to investigate whether the in vivo sound that results from the implant insertion hammer blows can be used to monitor the insertion process of cementless femoral implants. Sensitive vibro-acoustic features are identified in order to present a vibro-acoustic measurement method to monitor cementless implant seating.

\section{METHODS}

\section{Experimental protocol and set-up}

The acoustic response of the insertion hammer blows used to insert the implant in the host bone was measured during surgery. These insertion hammer blows are part of the standard surgical procedure and were applied on an inserter element that is in contact with the implant. This inserter element is part of the standard surgical equipment and is provided by the implant manufacturer. A condenser microphone (Type 378B02, PCB Piezotronics, Depew, NY, USA) was used to measure the acoustic response. A Scadas spectral analyzer (Siemens PLM, Leuven, Belgium) was used in combination with Simcenter Testlab software (Siemens PLM, Leuven, Belgium) as data acquisition system. The sampling frequency was $40.96 \mathrm{kHz}$.

An observational clinical feasibility study was conducted to assess the use of the method to monitor cementless femoral implant seating in an in vivo setting. Ethical approval for this study was granted by the Ethical Committee of KU Leuven/UZ Leuven. 26 patients (age range: 20 - 82 years) receiving a cementless femoral implant 
were included in this study. All 26 patients received a Profemur Gladiator implant (MicroPort Orthopedics, Arlington, TN, USA). All implants were inserted in the bone by using the corresponding inserters provided by the manufacturer. The acoustic response was measured by a microphone that was mounted on a mobile arm with internal cabling in order to ensure the sterility and was located at approximately $1 \mathrm{~m}$ from the bone-implant system. Figure 1a shows the microphone location in the operating theater, Figure $1 \mathrm{~b}$ shows a detailed view of the microphone and its connection to the mobile arm.

\section{Data processing}

A specific data processing approach was used to monitor the vibro-acoustic response of the insertion hammer blows. The raw acoustic time response data measured by the microphone was processed by isolating the individual insertion impacts and normalizing them to their maximum amplitude. Using the individual time response signals, the corresponding frequency spectra were calculated.

Subsequently, a specific set of data features was extracted from the response signals in order to monitor the changing conditions of the implant seating during the insertion procedure. Two types of data features were considered to monitor the implant seating; a first one based on the energy distribution in the frequency spectrum and a second one based on the shape of the frequency spectrum of the insertion hammer blows. The first type of feature, the band power feature (BPF), quantifies the relative spectral power distribution of the measured sound signal. The BPF is defined as the ratio of the power in a specific frequency band of interest over the total power of the frequency spectrum:

This article is protected by copyright. All rights reserved. 


$$
B P F=\frac{\sum_{f_{1}}^{f_{2}} P S D(f)}{\sum_{f_{1}}^{f_{3}} P S D(f)} * 100[\%]
$$

With,

$f_{1}=$ the lower frequency limit of the selected range $[\mathrm{Hz}]$,

$\mathrm{f}_{2}=$ the upper frequency limit of the range of interest $[\mathrm{Hz}]$,

$f_{3}=$ the upper frequency limit of the measurement range $[\mathrm{Hz}]$.

Figure 2 illustrates the frequency limits of the BPF concerning an example of an acoustic response spectrum.

A prior in vitro pilot study revealed that the spectral power in the lower frequency range is highly sensitive to implant insertions ${ }^{22}$. Following parameters were used in this study to calculate the BPF: $f_{1}=0.2 \mathrm{kHz}, \mathrm{f}_{2}=0.8 \mathrm{kHz}$ and $\mathrm{f}_{3}=20 \mathrm{kHz}$.

A second type of feature concentrates on the shape of the vibro-acoustic amplitude response spectra. Pearson correlation coefficients (PCC) were calculated as a distance metric between the vibro-acoustic response spectra of successive insertion hammer blows. These coefficients can be used as a measure of the similarity of two signals, varying between -1 (negative linear correlation) and 1 (positive linear correlation) and allows to quantify the changes in the vibro-acoustic response of the system over the insertion process. Three different Pearson correlation coefficients were calculated using the frequency response, which focus on three frequency zones: low (PCC 1), mid (PCC 2) and high (PCC 3). These metrics are calculated as the mean of the PCC(f) between successive frequency spectra in function of the upper limit of the frequency range (with variable f being the upper limit; and $0.2 \mathrm{kHz}$ always being the lower limit of the frequency range) as described by following relationship: 


$$
\overline{P C C(f)},=\frac{\Delta f}{f_{2}-f_{1}} \sum_{f_{1}}^{f_{2}} P C C(f)
$$

With $\Delta f$ the frequency resolution. This mean $\mathrm{PCC}(\mathrm{f})$ was calculated over the different frequency ranges: PCC $1\left(f_{1}=0.2 \mathrm{kHz}, f_{2}=0.8 \mathrm{kHz}\right), \operatorname{PCC} 2\left(f_{1}=0.2 \mathrm{kHz}, \mathrm{f}_{2}=1.5\right.$ $\mathrm{kHz})$ and PCC $3\left(\mathrm{f}_{1}=0.2 \mathrm{kHz}, \mathrm{f}_{2}=2.0 \mathrm{kHz}\right)$. This type of metric allows to reflect the overall similarity of the successive frequency spectra within the predefined range, without limiting the focus on one specific range.

A moving average of the BPF and PCC's was calculated using five impacts in order to reduce the influence of the environmental and operational variability that was inherent to the noise influences and the manual hammering procedure. All data processing was done using Matlab software (The MathWorks Inc., Natick, MA, USA). A paired sample t-test was used to compare the BPF between the initial and final phase of the insertion.

\section{RESULTS}

Out of the 26 implants that were inserted during the clinical study, 23 were inserted without any noticeable complications while during 3 insertions an intra-operative fracture occurred.

\section{Implant insertions without complications}

Figure 3 shows three case examples of the evolution of the BPF and PCC's obtained from measurements during implant insertion processes without complications. This pattern of increasing BPF and PCC's was observed in 18 out of the 23 implant insertions without complications (78\%), the BPF of these 18 cases increased on average $479 \%$ ( $p<<0.01)$. The BPF showed to be highly sensitive to the implant seating. Following observation demonstrated this sensitivity: a series of hammer 
blows during which the surgeon indicated that the implant subsided $1 \mathrm{~mm}$ displayed approximately a doubling of the BPF value. The increase of the low frequency content is clearly visible when comparing the frequency spectra of one of the first insertion hammer blows with one of the final hammer blows (Figure 4).

Aside the 18 cases that showed a consistent BPF pattern, there were 5 insertions without complications that showed an atypical evolution of the BPF. These cases did show an increase of the BPF but not as gradual as the other 18 cases and displayed an early convergence of the vibro-acoustic behavior characterized by a stationary trend of the BPF and PCC's. One of these cases was associated with suboptimal fixation due to a lack of proximal contact between the implant and bone.

\section{Intra-operative fractures}

Three intra-operative fractures were encountered during this clinical study. During the three implant insertions that were associated with intra-operative fractures, the BPF increased during the first part of the insertion process after which it dropped suddenly. A similar rapid decrease was observed in the evolution of the PCC's. Figure 5 shows the evolution of the BPF and PCC's during the insertion process of two fracture cases. These two insertion series were performed without pauses and by a consistent hammering direction, which minimizes the operational variability.

\section{DISCUSSION}

The sensitivity of the BPF to the insertion process can be explained by the increasing level of fixation of the implant in the femur. During the initial phase of the insertion, when no adequate fixation between implant and bone exists, the boundary conditions of the implant-inserter system are comparable to free-free conditions. These conditions result in frequency content dominated by the higher modes of the implant-

This article is protected by copyright. All rights reserved. 
inserter system. As the insertion progresses, the implant starts to bond with the host bone due to the increasing contact area. This leads to a shift of the assumed free-free conditioned implant-inserter system to a bonded bone-implant-inserter system resulting in a more efficient force transfer between implant and bone. This increasing bond introduces low frequency content related to the lower modes of the combined bone-implant-inserter system. Another influence on the BPF may be related to the changing damping conditions as a result of the increasing contact area between implant and bone as the insertion process progresses. The increasing contact area between implant and bone introduces supplementary damping in the system. Higher frequency modes are prone to this elevated damping, resulting in a reduction of this content in the frequency spectrum. The combination of the above-mentioned effects results in a shift of the spectral power from the higher to the lower frequency ranges, as reflected by the BPF that characterizes the ratio of low frequency power compared to the power present in the entire frequency spectrum. This effect is also visible when comparing the frequency spectra of one of the first hammer blows with one of the final hammer blows during the implant insertion (Figure 4). This shift from high to low frequency content is in correspondence with previous studies that found similar increase of the low frequency content during broach and implant insertion experiments ${ }^{13-15,18-21} .{ }^{13}$ allocated this low frequency increase to the development of a standing wave in the femoral canal as a result of the increasing bond between broach and bone. ${ }^{14}$ found a $449 \%$ increase in a low frequency band power during broaching experiments, ${ }^{19}$ revealed a decreasing main frequency peak as a femoral implant was inserted in an artificial bone model and ${ }^{18}$ mentioned in a cadaveric case study the appearance of low frequency components as a last broach was inserted. Most of these studies revealed this tendency of a change in low frequency behavior of the acoustic This article is protected by copyright. All rights reserved. 
response in vitro, this study demonstrated that these findings were also observable in a more demanding and less controlled in vivo setting (even with the addition of environmental noise and additional soft tissue damping).

The BPF showed considerable variation at the end of the insertion over the different surgeries (coefficient of variation of $47 \%$ ). Differences in the studied systems, environmental and operational variability are most likely the causes of the rather high variation on the feature values towards the end of the insertion. The differences in the studied systems are related to the different inserters and hammers and to patient variability such as soft tissue coverage, fluid interactions and bone properties. The operator variability is related to the differences in hammering direction, inserter orientation and contact between the inserter and the soft tissue of the patient. The environmental variability is related to environmental noise that is present in the surgical theater, which affects the signal-to-noise ratio (SNR). Similarly, the PCC's towards the end of the insertion showed some degree of variation between the different surgeries, which is mainly due to differences in the SNR's. Lower SNR likely lead to a lower correlation coefficient between comparable signals due to the random effect of the added noise.

Several insertions displayed a reduction of the BPF after a pause in the insertion process (varying between approximately 30 and 60 seconds), as shown for one example in Figure 6. The orthopaedic surgeon included these pauses in the insertion process in order to allow stress relaxation to occur in the bone, which is reported to occur within this time range after stress is applied ${ }^{23}$. This stress relaxation in the bone could be the reason for the observed reduction of the BPF.

This article is protected by copyright. All rights reserved. 
The insertion series that were associated with intra-operative fractures showed a decrease of the BPF and PCC's at a specific point during the insertion series. The sudden change of the trend of the BPF and PCC's started around step 20 in both examples of Figure 5. This abrupt change could be initiated by the occurrence of a micro or macro fracture. A fracture results in a sudden stress release in the bone surrounding the implant leading to a decrease of the press-fit of the implant. This decrease of press-fit and corresponding implant fixation is translated in a substantial change of the vibro-acoustic response as reflected by the drop of the BPF and correlation coefficients as observed in these measurements. Especially during the first example presented in Figure 5, the BPF dropped radically (approximately 75\%) halfway the insertion process towards a value that is associated with the initial phase of the insertion that corresponds to a loose implant. Even though the vibro-acoustic behavior showed a radical change halfway this insertion process, 15 more hammer blows were applied after this change until the surgeon noticed the fracture. These findings show the potential of using a drastic drop of the BPF and PCC's within a consistent and continuous hammering series as an indicator of loss of implant stability, which should be considered as a warning for the occurrence of a periprosthetic (micro)fracture.

\section{Convergence-based endpoint criterion}

Given the high sensitivity of the BPF and PCC's to the insertion process as similarly observed during prior in vitro experiments ${ }^{22}$, a specific convergence based criterion is proposed in order to create an objective method to identify a candidate endpoint of the insertion based on the acoustic response of the bone-implant system. This criterion is based on the convergence of the vibro-acoustic behavior that is observed towards the

This article is protected by copyright. All rights reserved. 
end of the insertion process, which is associated with reaching the maximal implant fixation. This principle originates from the orthopaedic surgeons who use acoustic cues as source of information on the implant seating and is confirmed by prior in vitro experiments ${ }^{22}$. Using the data of the implant insertion measurements that showed a gradually increasing $\mathrm{BPF}$, an endpoint detection criterion is proposed that is based on the combination of the previously described BPF and correlation coefficients related to the frequency signal. The criterion used two parameters to define the convergence of a feature or metric: a number of preceding steps to compare the feature/metric values and coefficient of variation $(\mathrm{COV})$. These parameters defined the strictness of convergence. Based on results of training data, initial parameter values were determined during this study. The convergence is defined as the moment when the $\mathrm{COV}$ of the feature/metric values of the three most recent insertion steps was less than $5 \%$ and the final feature value lied within this 5\% interval. This approach was applied for the BPF and different PCC's. Additional convergence criteria were that the BPF converged, as the BPF showed to be the most sensitive feature to changing boneimplant contact and that the PCC's were higher than 0.6 in order to ensure the consideration of similar signals. The number of converged features or metrics at a specific insertion step defined the corresponding level of convergence (denoted as convergence index (CI), varying between 1 and 4). Using this information, a high degree of convergence in an advanced phase of the insertion process could indicate a possible endpoint insertion as it reflects stationary vibro-acoustic behavior. Figure 7 illustrates how convergence is defined using the combined information of the BPF and the PCC's of the case examples of Figure 3. The convergence of a single feature or metric is indicated by the green circle, while the overall level of convergence per step is indicated by the triangles on the x-axis. A black, yellow (does not occur in the 
presented examples), blue or green triangle denotes a convergence index of 1, 2, 3 or 4 respectively. In the examples of Figure 7, the green triangles mark the moment at which the BPF and all PCC's converged during that implant insertion process according to this convergence criterion. The performance of the convergence criterion is assessed by comparing the moment of advanced convergence of vibro-acoustic behavior to the endpoint as it was determined by the surgeon. The surgical endpoint is defined by the orthopaedic surgeon as the moment at which maximal implant stability is reached.

The end of the insertions presented in Figure 7 (final step on the $\mathrm{x}$-axis) corresponds to the endpoint of insertion as determined by the orthopaedic surgeon. The application of the criterion indicated a strong convergence of the vibro-acoustic behavior towards the final phase of the insertion process as marked by the green triangle(s). Overall, the criterion indicated an advanced level of convergence $(\mathrm{CI}>3)$ near the endpoint as determined by the surgeon for 17 out of the 23 insertion cases without complications (74\%). The remaining cases showed an advanced degree of converged vibro-acoustic behavior early in the insertion process. One of these cases was associated with insufficient implant fixation due to improper bone-implant contact in the proximal region, as stated earlier.

Some general remarks need to be addressed regarding the presented study and measurement method. A number of parameters to calculate the presented BPF and PCC's were chosen empirically during the presented study. It is important to state that these parameters should not be perceived as the sole or optimal parameters, but form a sound basis for this analysis. The upper limit of the frequency range to calculate the BPF of the femoral implant insertions $(800 \mathrm{~Hz})$ was based on the comparison of the 
different response spectra in combination with the rationale that the response of the lower femur modes are the cause of the increase in BPF. Earlier in vitro experiments and in silico studies showed that the first two bending modes of the femur-implant system exist in the range of approximately $200 \mathrm{~Hz}-800 \mathrm{~Hz}^{24}$. The results deteriorated when increasing the upper range; some cases did not show convergence or the sensitivity decreased considerably. Figure 8 illustrates case examples of the influence of different frequency ranges on the normalized BPF during the implant insertion. Increasing the upper limit of the BPF resulted in a decrease of the sensitivity of the BPF to the implant insertion process.

The number of PCC's (3) and corresponding upper ranges $(800 \mathrm{~Hz}, 1500 \mathrm{~Hz}$ and 2000 $\mathrm{Hz}$ ) were chosen to cover the behavior within three regions in the low frequency range based on the low frequency sensitivity of the BPF to the implant seating. Upper limits up to $2000 \mathrm{~Hz}$ showed insertion sensitive results. When considering upper limits above $2000 \mathrm{~Hz}$, the sensitivity of the PCC to the insertion process reduced considerably as illustrated in Figure 9. This analysis of the sensitivity of both the BPF and the PCC's confirms once more the importance of the low frequency behavior of the acoustic response when monitoring the implant seating.

The BPF and PCC's showed to be sensitive to the implant insertion but did also show a dependency on external noise disturbance and operational variability such as irregularities in the hammering process. Environmental noise sources (e.g. speech or noise of the suction tool) may lead to a momentary decrease of the PCC and in some cases a rise of the BPF (depending on its frequency content). Similar effects were observed when the surgeon changed the inserter or hammering orientation during the insertion. These effects could lead to false negatives when considering a convergence 
of the vibro-acoustic behavior. Hence, efforts to increase the SNR for example by closer positioning the microphone to the surgical work field to reduce the negative influence of unrelated sound sources and a consistent insertion procedure (hammer and inserter orientation) offer a solution to avoid these adverse effects.

This in vivo study has some limitations that need to be addressed. First, the surgeon's judgement is used as a reference regarding the optimal endpoint. Since a stability metric in terms of bone-implant micromotion measurements is not feasible in an in vivo study, a follow-up study could provide additional information regarding the longterm stability of the implant. Especially for the implant insertion cases that showed an atypical evolution of the BPF and PCC's, a comparison with the long term outcome of the implant could be interesting to see if there exists a correlation with adverse events such as excessive subsidence or indications of early loosening. However, the main goal of this in vivo study was to assess the evolution and convergence behavior of the different features over the insertion process.

\section{CONCLUSION}

A real-time vibro-acoustic measurement method is presented to monitor the insertion of cementless THA implants based on the analysis of the insertion hammer blows. The vibro-acoustic response of the insertion of cementless THA implants was measured during 26 in vivo implant insertions. Cementless femoral implant insertions were studied and resulted in sensitive low-frequency behavior, confirming the results observed in prior in vitro experiments. A convergence-based endpoint criterion was used to identify stationary behavior of the sensitive vibro-acoustic features. The convergence of the sensitive vibro-acoustic behavior agreed well with the endpoint of insertion as determined by the orthopaedic surgeon.

This article is protected by copyright. All rights reserved. 
Three intra-operative fractures were encountered during the in vivo study on the femoral stem insertions. During these implant insertions, the vibro-acoustic response showed an abrupt decrease of the low-frequency content. This abrupt change of the vibro-acoustic behavior occurred prior to the notification of the fracture by the orthopaedic surgeon and thus could serve as an important early warning for severe loss of implant stability.

Acoustic approaches have been investigated for post-operative assessment of implant loosening ${ }^{25}$. Complementary to these post-operative methods, the presented method focuses on the intra-operative assessment of implant fixation. A solely acoustic approach is proposed, which has major advantages towards the implementation in a clinical setting. No measurement equipment needs to be attached to the bone-implant system, which reduces the risk of infections and omits an obstruction of the surgical workspace. Previous research explored the use of an alternative acoustic method during which light non-destructive impacts are applied at specific stages during the insertion process ${ }^{26}$. In contrast, the presented method uses the standard surgical hammer blows as excitation. Inherent to this type of excitation is the important advantage that it allows to monitor the behavior continuously without the need of extra actions by the surgeon. This prevents unnecessary time loss during the operation.

A disadvantage of the acoustic method is that environmental noise and operational variability may influence the measurement outcomes. In order to maximize the sensitivity of the method, it is recommended to average the data (as applied in this study) but also to minimize the environmental and operational variability; care should be taken to avoid excessive environmental noise and to apply a consistent hammering 
series to insert the implant. In contrast, other research focused on contact-based vibrational measurement methods in vitro ${ }^{27-31}$ and in vivo ${ }^{12}$. Although these contactbased methods have the advantage to produce precise results and are more robust against environmental and operational variabilities, they possess the disadvantage of requiring sensors to be attached to the bone or implant in combination with the use of an instrumented excitation. This contact requirement introduces an additional risk for infections. Additionally, these sensors or actuators can obstruct the surgeon's workspace and require to be mounted and dismounted which leads to undesired time loss during the operation.

The intent of the presented measurement method is not to replace the current judgement and decisions of the orthopaedic surgeon, but to serve as a decisionsupporting source of information regarding the evolution of the vibro-acoustic response of the bone-implant system as it showed to reflect the implant seating. The presented method can be packaged into a compact medical device that comprises one or more microphones, a data processing unit and a user interface that can be placed conveniently in the surgical theater (e.g. attached to a surgical lamp). The use of this device can provide real-time feedback on a set of vibro-acoustic features that serve as an additional source of information on the implant stability to support the surgeon's judgement.

\section{ACKNOWLEDGEMENTS}

This research was supported by Internal Funds KU Leuven.

This article is protected by copyright. All rights reserved. 


\section{REFERENCES}

1. Orthopride. 2017. Belgian Hip and Knee Arthroplasty Registry: Annual Report 2015-2016. [cited 2019 Jun 20].

2. Gheduzzi S, Miles AW. 2007. A review of pre-clinical testing of femoral stem subsidence and comparison with clinical data. Proceedings of the Institution of Mechanical Engineers, Part H: Journal of Engineering in Medicine 221(1):39-46.

3. Pilliar RM, Lee JM, Maniatopoulos DDS. 1986. Observations on the Effect of Movement on Bone Ingrowth into Porous-Surfaced Implants. Clinical Orthopaedics and Related Research 208:108-113.

4. Jasty M, Bragdon C, Burke D, et al. 1997. In Vivo Skeletal Responses to PorousSurfaced Implants Subjected to Small Induced Motions*. The Journal of Bone and Joint Surgery (American Volume) 79(5):707-714.

5. Meek RMD, Garbuz DS, Masri BA, et al. 2004. Intraoperative Fracture of the Femur in Revision Total Hip Arthroplasty with a Diaphyseal Fitting Stem: The Journal of Bone \& Joint Surgery 86(3):480-485.

6. Lindahl H. 2007. Epidemiology of periprosthetic femur fracture around a total hip arthroplasty. Injury 38(6):651-654.

7. Tsiridis E, Haddad FS, Gie GA. 2003. The management of periprosthetic femoral fractures around hip replacements. Injury 34(2):95-105.

8. Pastrav LC, Jaecques SVN, Mulier M, Van der Perre G. 2008. Detection of the Insertion End Point of Cementless Hip Prostheses using the Comparison between Successive Frequency Response Functions. Journal of Applied Biomaterials \& Biomechanics 6(1):23-29.

9. Crisman A, Yoder N, McCuskey M, et al. 2007. Femoral Component Insertion Monitoring Using Human Cadaveric Specimens. In: IMAC Proceedings..

10. Cruce J, Erwin J, Remick K, et al. 2011. Peri-Prosthetic Fracture Vibration Testing. In: Sensors, Instrumentation and Special Topics, Volume 6. New York, NY: Springer New York. p 207-225.

11. Cachão JH, Soares dos Santos MP, Bernardo R, et al. 2019. Altering the Course of Technologies to Monitor Loosening States of Endoprosthetic Implants. Sensors 20(1):104.

12. Pastrav LC, Jaecques SVN, Jonkers I, et al. 2009. In vivo evaluation of a vibration analysis technique for the per-operative monitoring of the fixation of hip prostheses. Journal of Orthopaedic Surgery and Research 4(1):10.

13. Whitwell G, Brockett CL, Young S, et al. 2013. Spectral analysis of the sound produced during femoral broaching and implant insertion in uncemented total hip arthroplasty. Proceedings of the Institution of Mechanical Engineers, Part H: Journal of Engineering in Medicine 227(2):175-180.

This article is protected by copyright. All rights reserved. 
14. Abdulkarim A, Jackson B, Riordan D, Rice J. 2013. The use of sound analysis to guide femoral reaming in uncemented total hip arthroplasty: A new concept. International Journal of Surgery 11(8):593.

15. Paech A, Cabrera-Palacios H, Schulz A, et al. 2008. Acoustic Tests on Hip Prosthesis Models Using Frequency Resonance Monitoring (FRM). Research Journal of Medical Sciences 2(2):82-91.

16. Unger A, Cabrera-Palacios H, Schulz A, et al. 2009. Acoustic monitoring (RFM) of total hip arthroplasty results of a cadaver study. European Journal of Medical Research 14(6):264.

17. Paech A, Cabrera-Palacios H, Schulz AP, et al. 2007. Acoustic Properties of Femoral Components of Hip Endoprostheses Analysing Using FrequencyResonance-Measurement in a Soft Tissue Simulation Model. Research Journal of Medical Sciences 1(2):118-123.

18. Oberst S, Baetz J, Campbell G, et al. 2018. Vibro-acoustic and nonlinear analysis of cadavric femoral bone impaction in cavity preparations. MATEC Web of Conferences 148:14007.

19. Sakai R, Kikuchi A, Morita T, et al. 2011. Hammering sound frequency analysis and prevention of intraoperative periprosthetic fractures during total hip arthroplasty. Hip International 21(6):718-723.

20. Oyama J, Furuichi I, Murata M, Endo M. 2013. Sound Wave Analysis of Cementless Hip Stem Hammering. Orthopaedic Proceedings 95-B(15):297-297.

21. Morohashi I, Iwase H, Kanda A, et al. 2017. Acoustic pattern evaluation during cementless hip arthroplasty surgery may be a new method for predicting complications. SICOT-J 3:13.

22. Goossens Q, Leuridan S, Roosen J, et al. 2015. Monitoring of reamer seating using acoustic information. In: Annual meeting of the European Society of Biomechanics. Prague.

23. Zilch H, Rohlmann A, Bergmann G, Kol R. 1980. Material properties of femoral cancellous bone in axial loading. Archives of Orthopaedic and Trauma Surgery 97:257-262.

24. Pastrav LC, Devos J, Van der Perre G, Jaecques SVN. 2009. A finite element analysis of the vibrational behaviour of the intra-operatively manufactured prosthesis-femur system. Medical Engineering \& Physics 31(4):489-494.

25. Ruther C, Gabler C, Ewald H, et al. 2014. In vivo monitoring of implant osseointegration in a rabbit model using acoustic sound analysis. Journal of Orthopaedic Research 32(4):606-612.

26. Goossens Q, Leuridan S, Henyš $\mathrm{P}$, et al. 2017. Development of an acoustic measurement protocol to monitor acetabular implant fixation in cementless total hip Arthroplasty: A preliminary study. Medical Engineering \& Physics 49:28-38.

This article is protected by copyright. All rights reserved. 
27. Leuridan S, Goossens Q, Vander Sloten T, et al. 2017. Vibration-based fixation assessment of tibial knee implants: A combined in vitro and in silico feasibility study. Medical Engineering \& Physics 49:109-120.

28. Lannocca M, Varini E, Cappello A, et al. 2007. Intra-operative evaluation of cementless hip implant stability: A prototype device based on vibration analysis. Medical Engineering \& Physics 29(8):886-894.

29. Alshuhri AA, Holsgrove TP, Miles AW, Cunningham JL. 2015. Development of a non-invasive diagnostic technique for acetabular component loosening in total hip replacements. Medical Engineering \& Physics 37(8):739-745.

30. Henyš P, Capek L, Fencl J, Prochazka E. 2015. Evaluation of acetabular cup initial fixation by using resonance frequency principle. Proceedings of the Institution of Mechanical Engineers, Part H: Journal of Engineering in Medicine 229(1):3-8.

31. Tijou A, Rosi G, Vayron R, et al. 2018. Monitoring cementless femoral stem insertion by impact analyses: An in vitro study. Journal of the Mechanical Behavior of Biomedical Materials 88:102-108.

\section{FIGURES}

Figure 1. The clinical set-up of the microphone to record the acoustic response (a). The microphone was mounted on a mobile arm with internal cabling in order to ensure the sterility (b). The microphone was located at approximately $1 \mathrm{~m}$ from the bone-implant system zone.

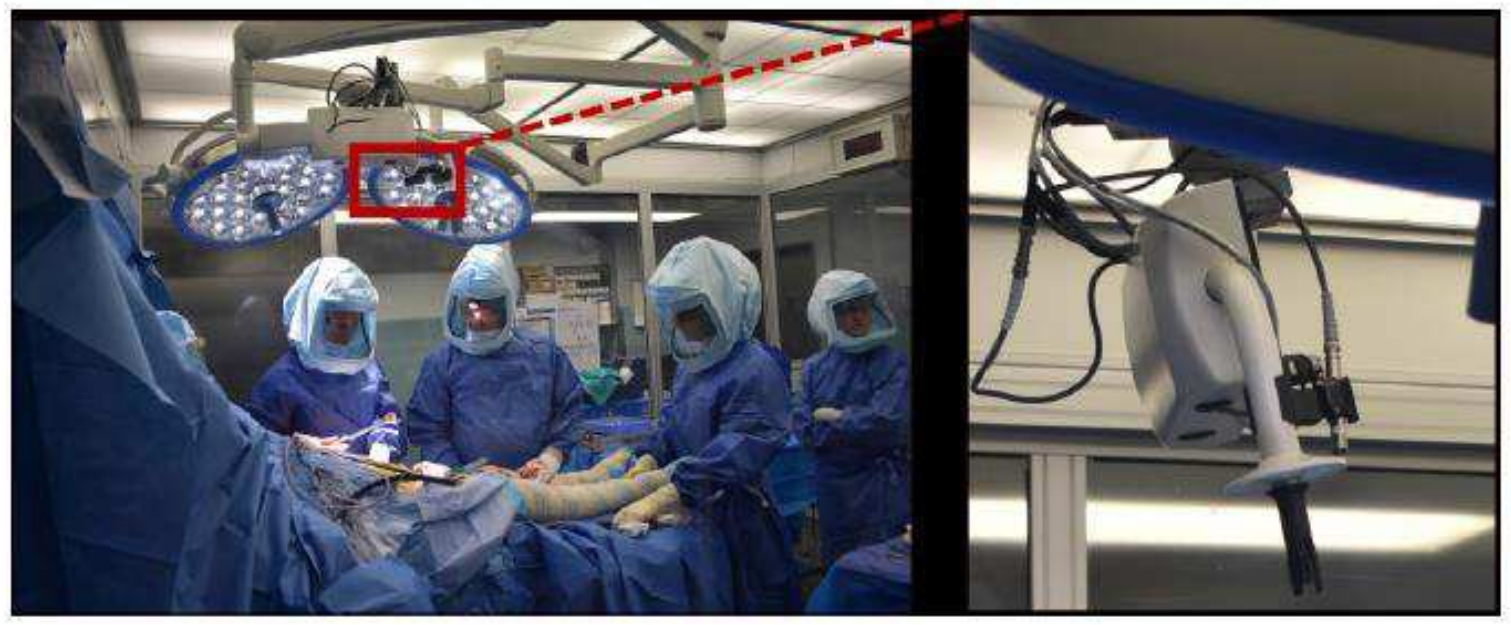

This article is protected by copyright. All rights reserved. 
Figure 2 Illustration of the frequency limits $f 1, f 2$ and $f 3$ used to calculate the BPF.

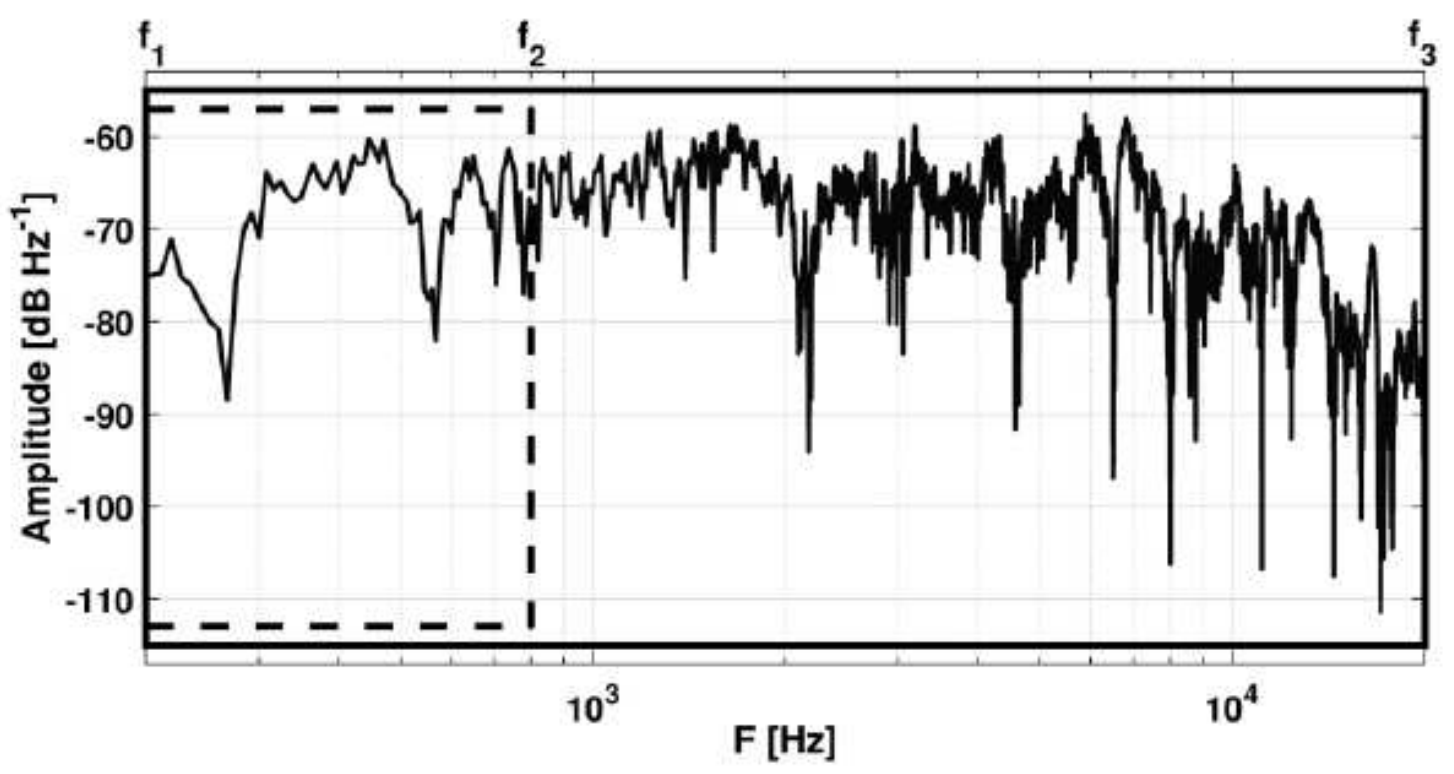

Figure 3 Case examples of the evolution of the BPF and PCC's measured over the insertion process of a femoral implant without any complication.

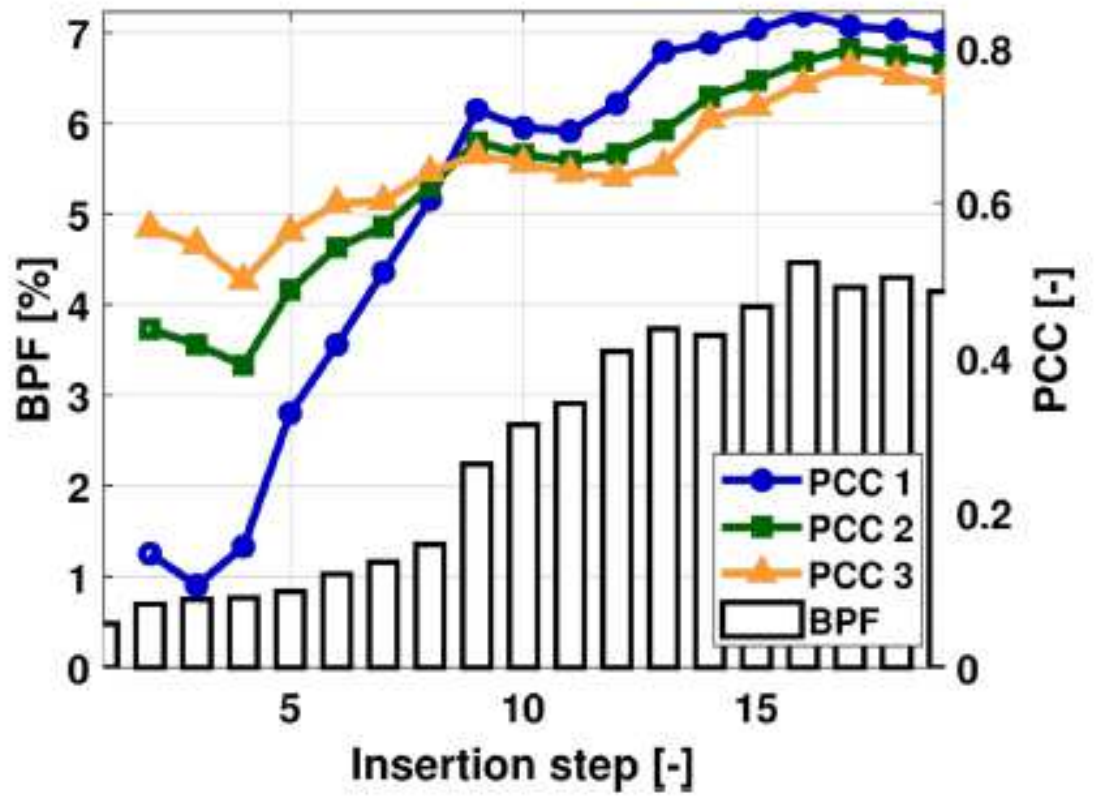

This article is protected by copyright. All rights reserved. 

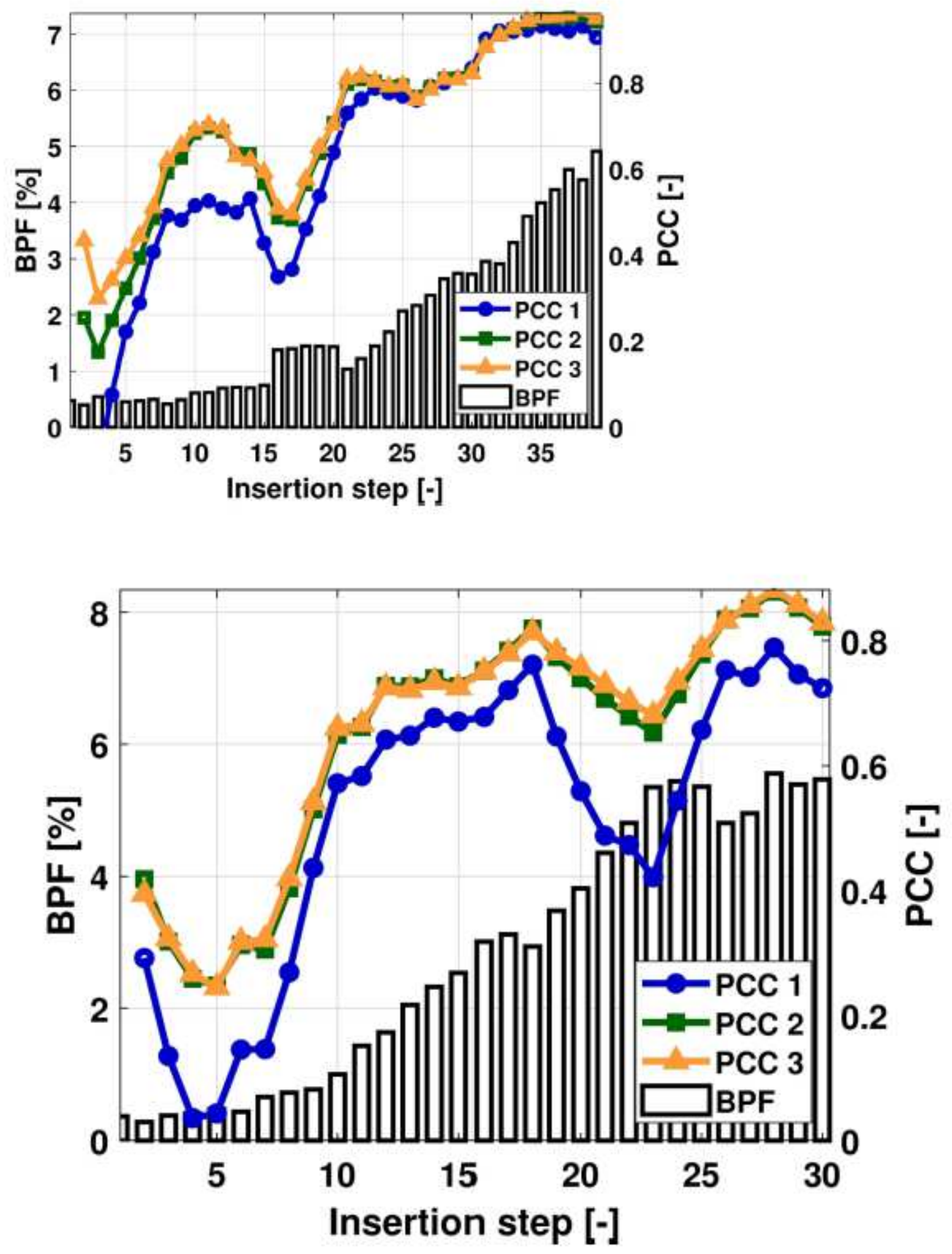

This article is protected by copyright. All rights reserved. 
Figure 4 An example of the spectra obtained from the first (gray line) and final (black line) insertion hammer blows measured during one of the femoral implant insertions.

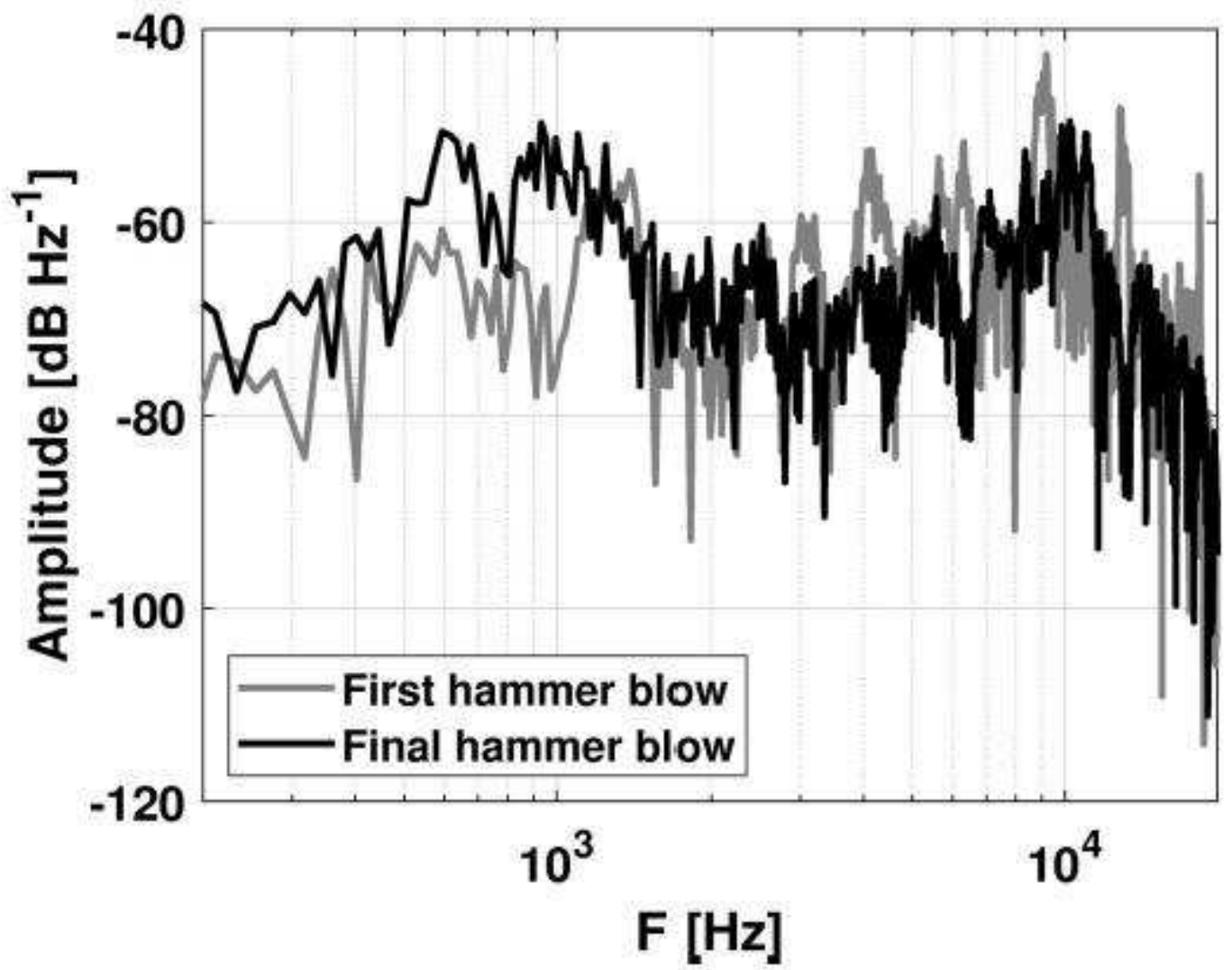

Figure 5 Two case examples of the evolution of the BPF and PCC's measured over the insertion process of a femoral implant during which an intra-operative fracture occurred. The change in the BPF and PCC evolution is marked by the red box.

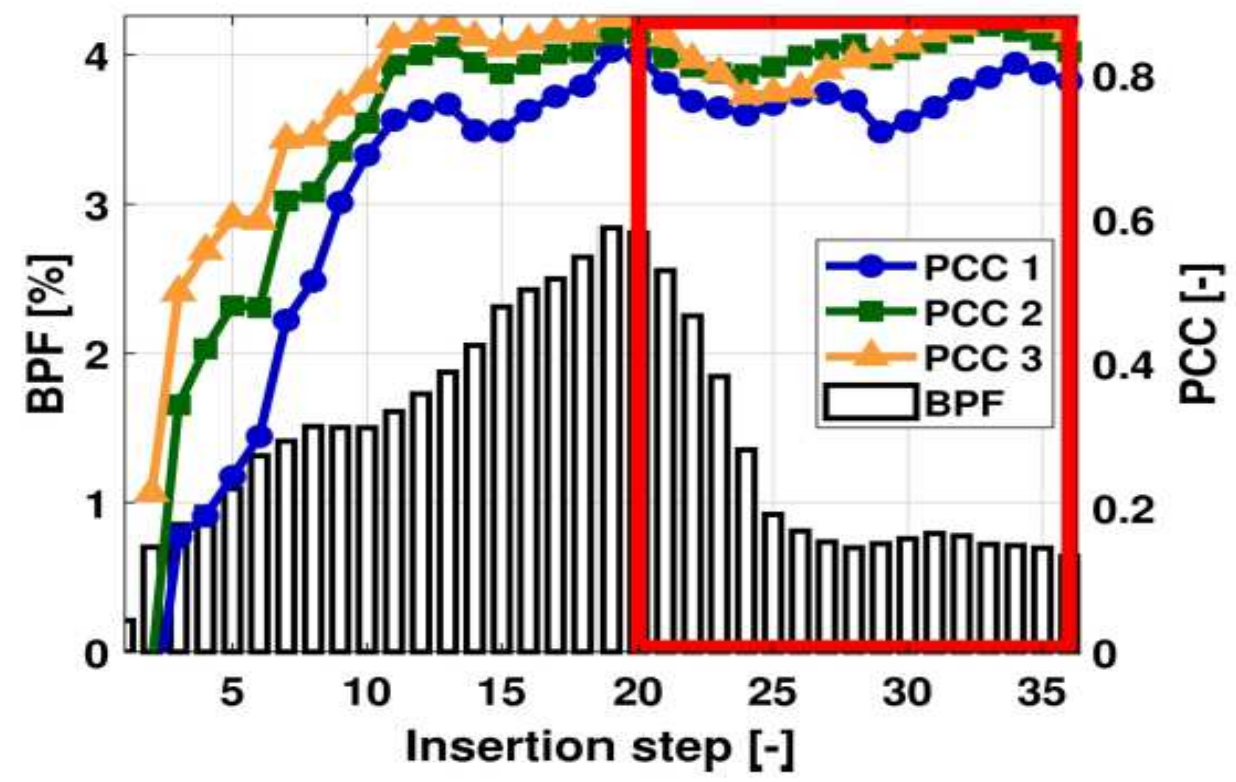

This article is protected by copyright. All rights reserved. 


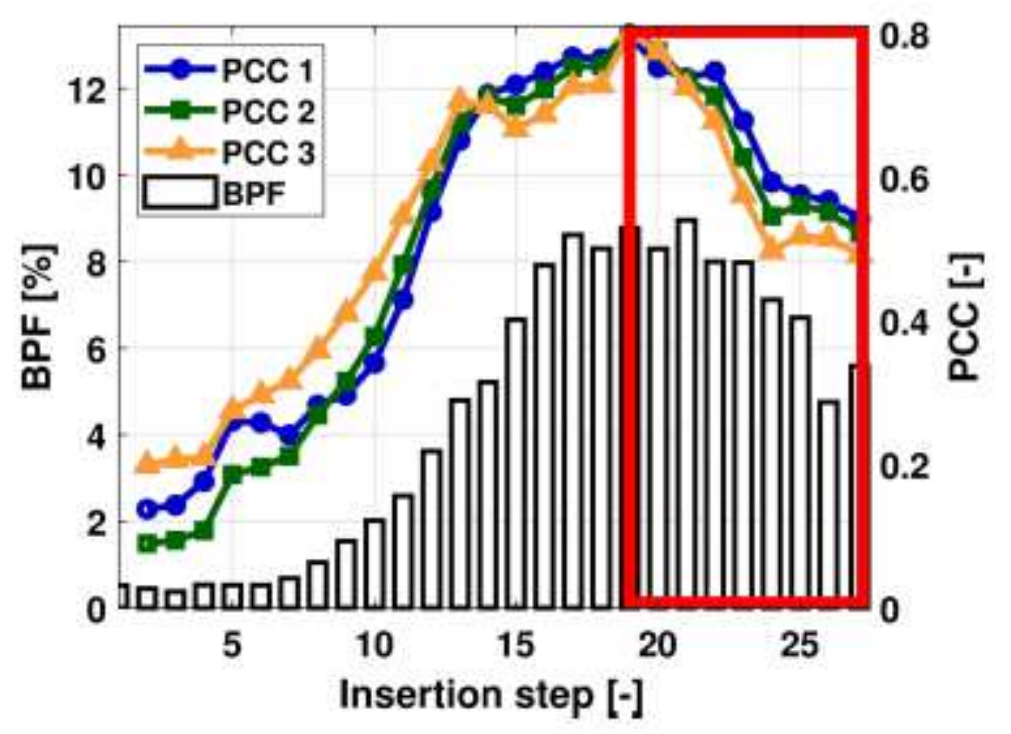

Figure 6 Case example to illustrate the reduction of the BPF after a pause marked by the arrow. The duration of the pause corresponding to the marked drop was $60 \mathrm{~s}$.

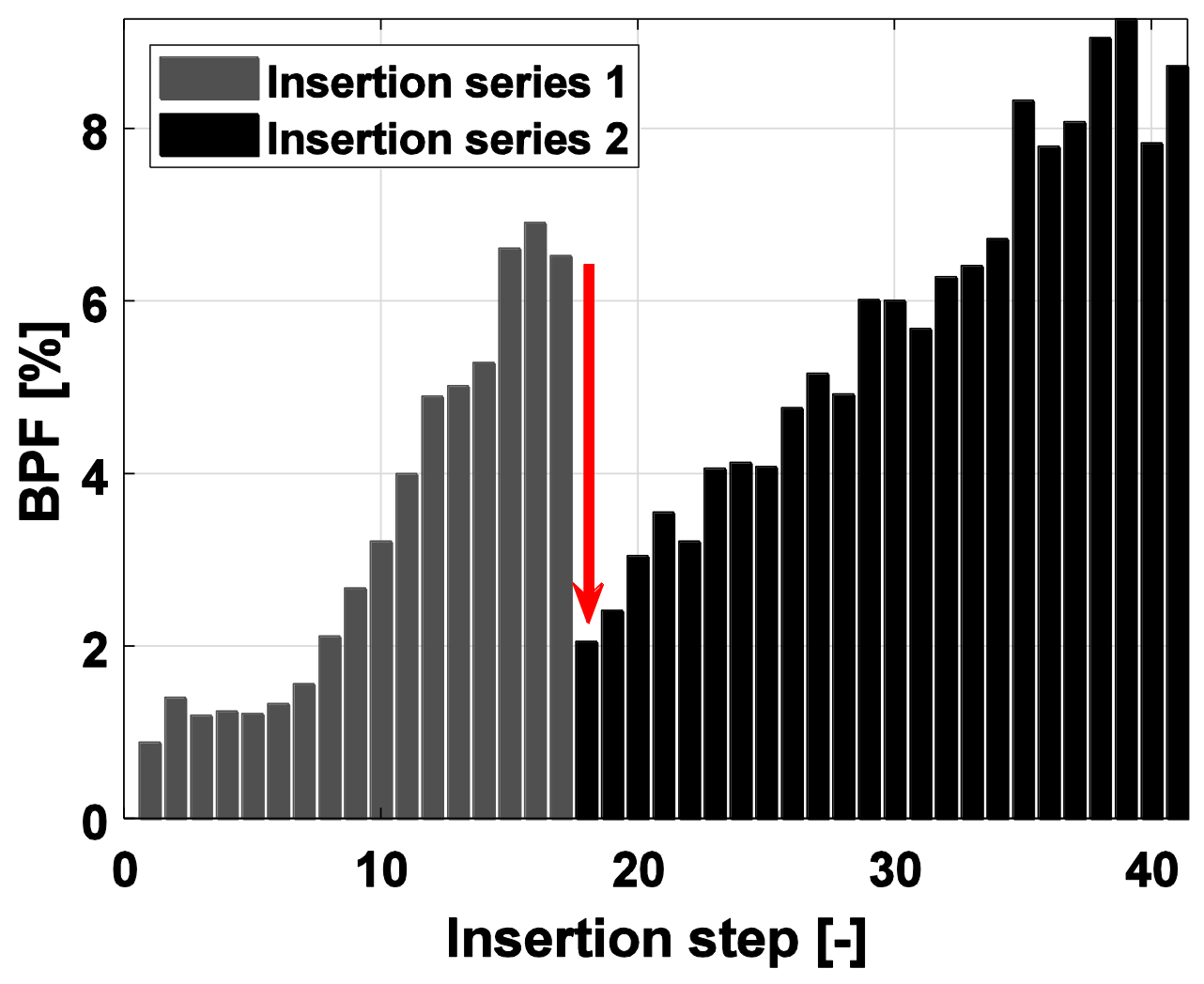

This article is protected by copyright. All rights reserved. 
Figure 7 Illustration of the convergence-based endpoint criterion based on the evolution of the different features during an insertion process. The black triangles denote the insertion steps at which 1 feature or metric converged, the blue triangles denote the insertion steps at which 3 features or metrics converged and the green triangles denote the insertion steps at which 4 features or metrics converged.
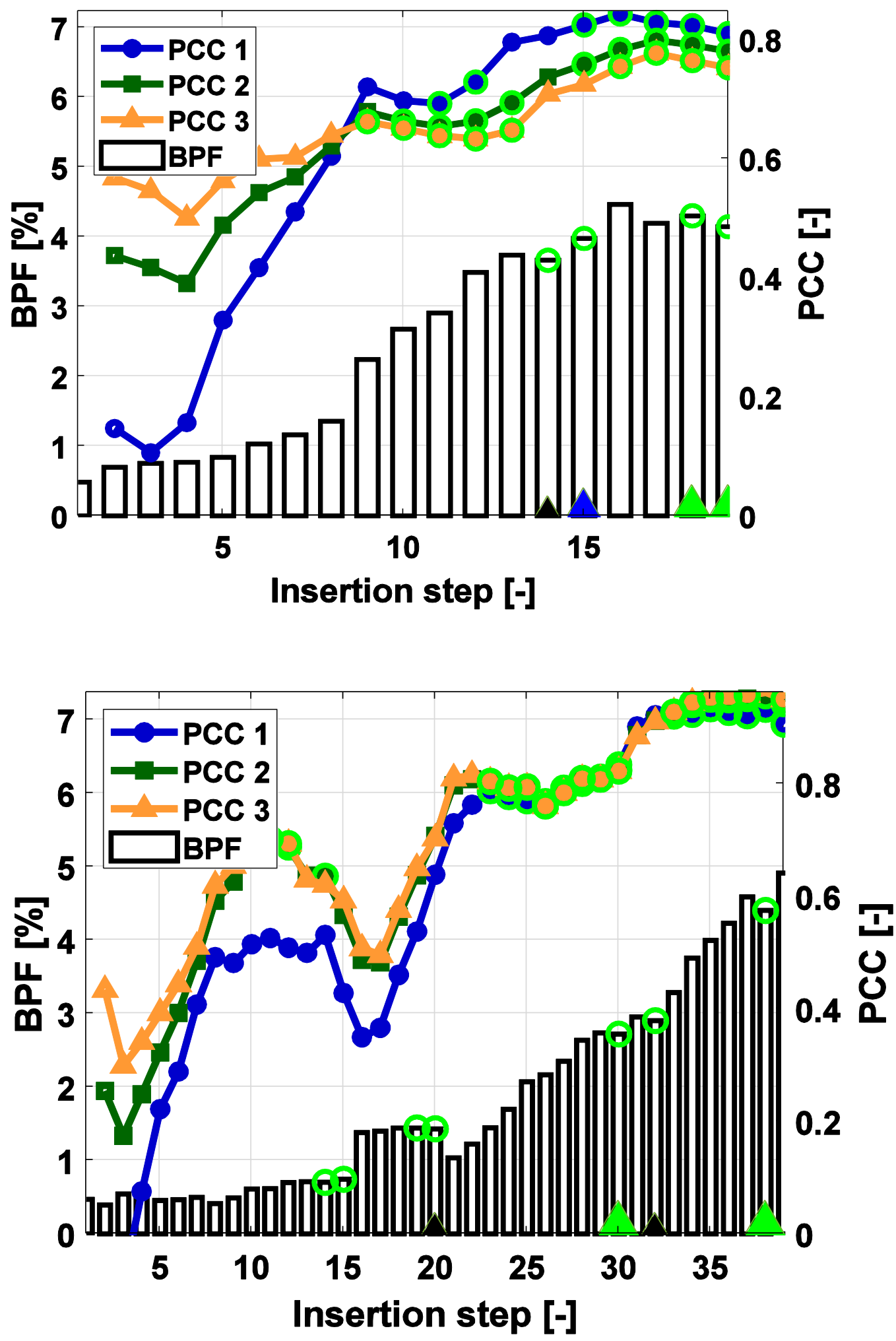

This article is protected by copyright. All rights reserved. 


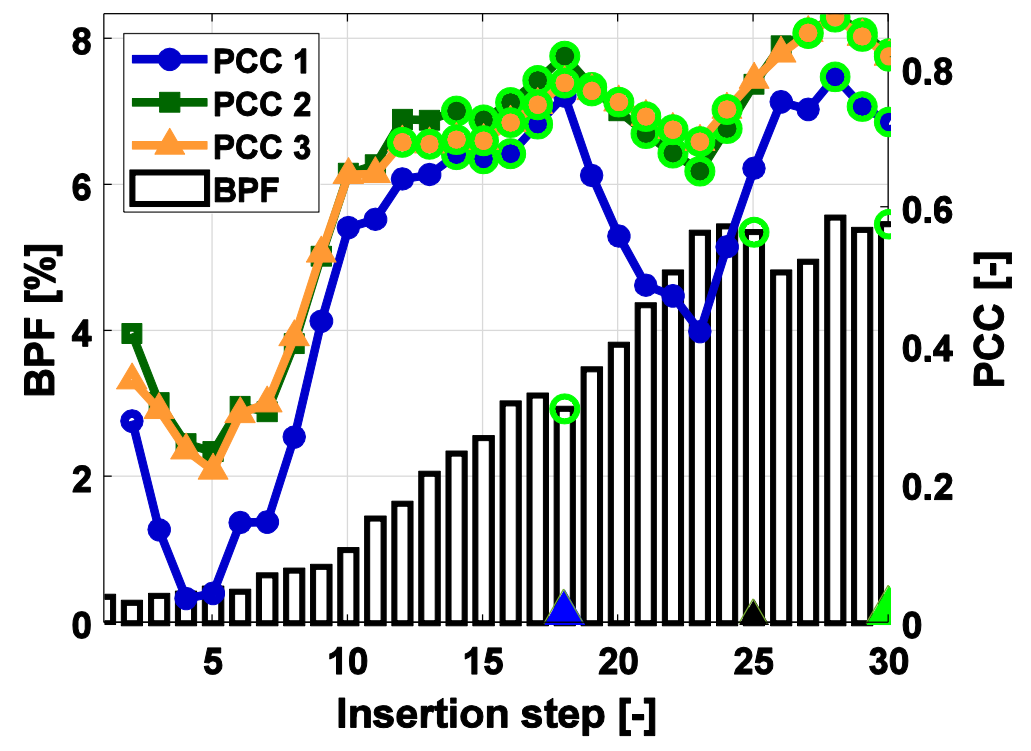

Figure 8 Case examples of femoral implant insertions to illustrate the sensitivity of the BPF when using different frequency ranges.

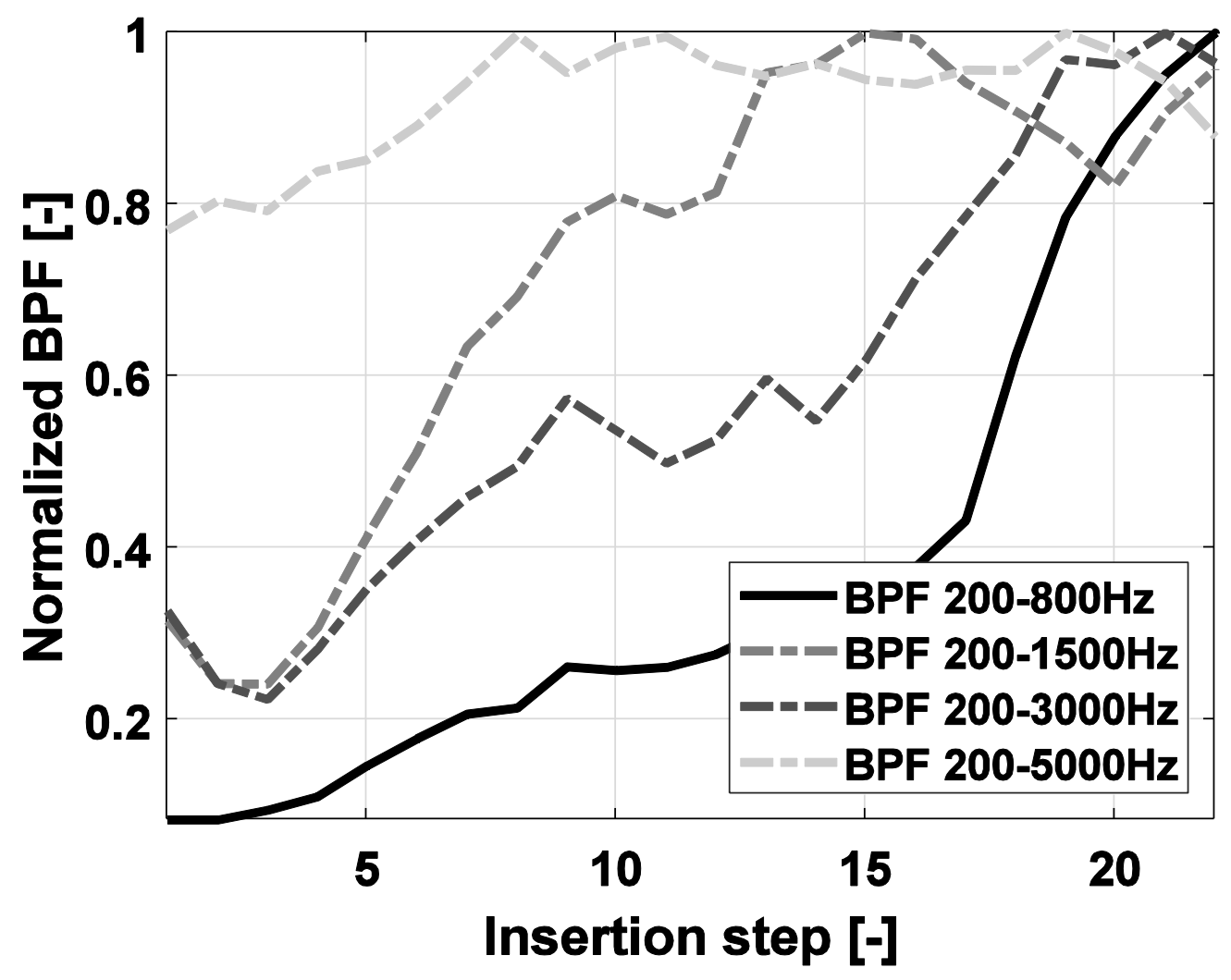

This article is protected by copyright. All rights reserved. 


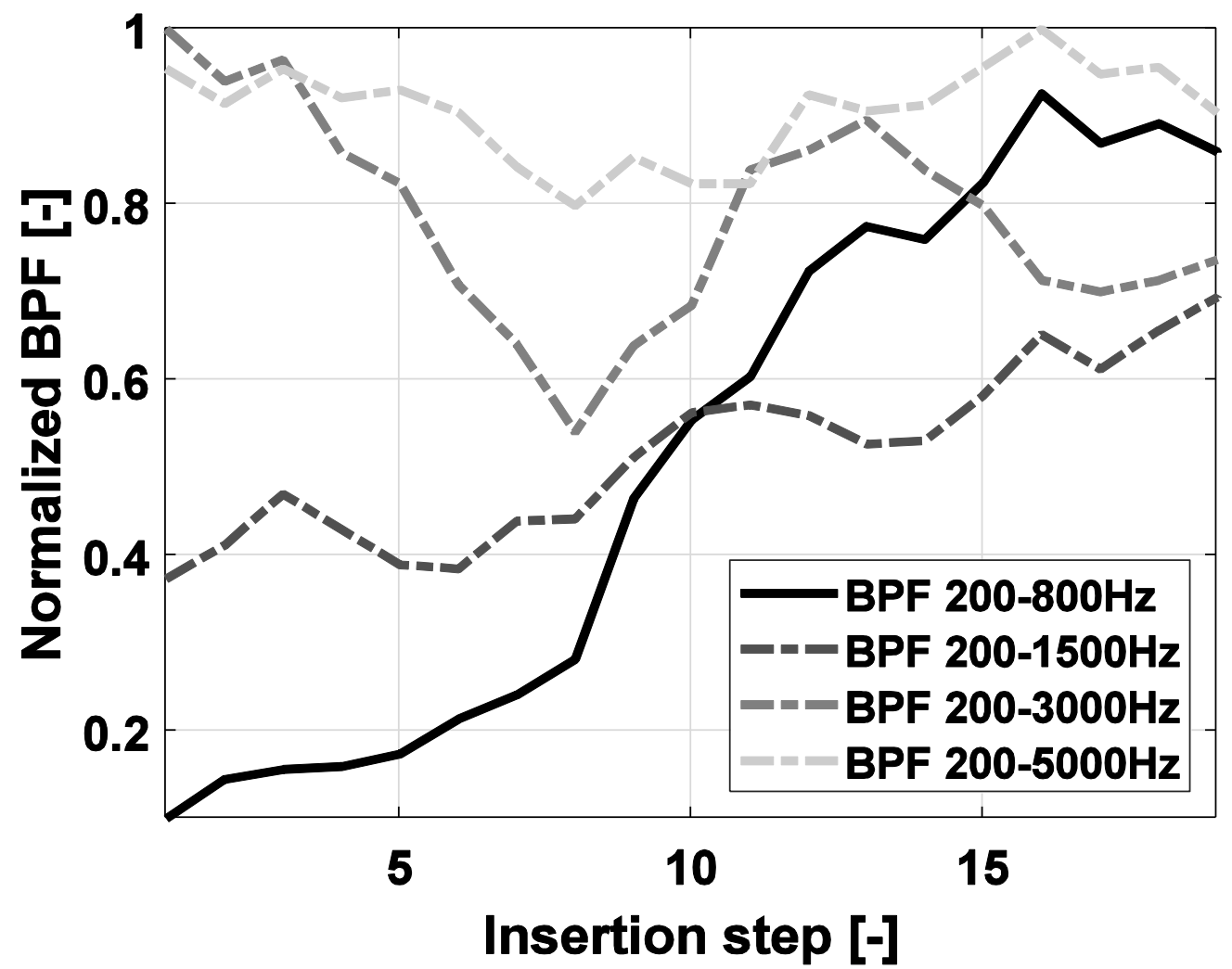

Figure 9 Case examples of femoral implant insertions to illustrate the sensitivity of the PCC's when using different frequency ranges.

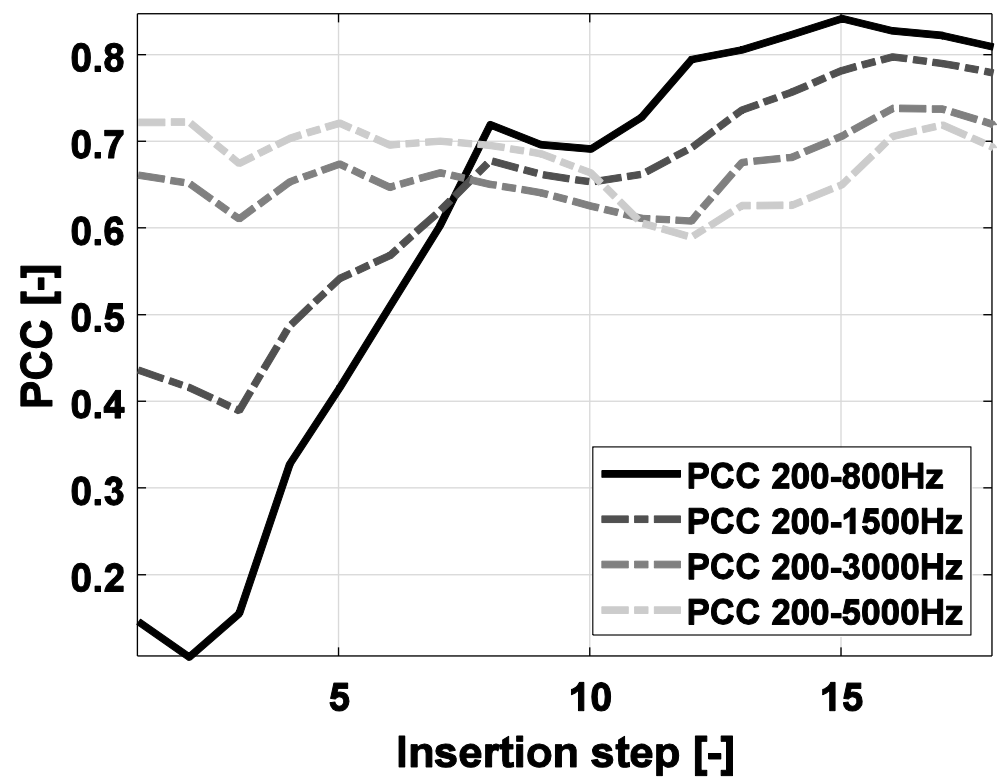

This article is protected by copyright. All rights reserved. 


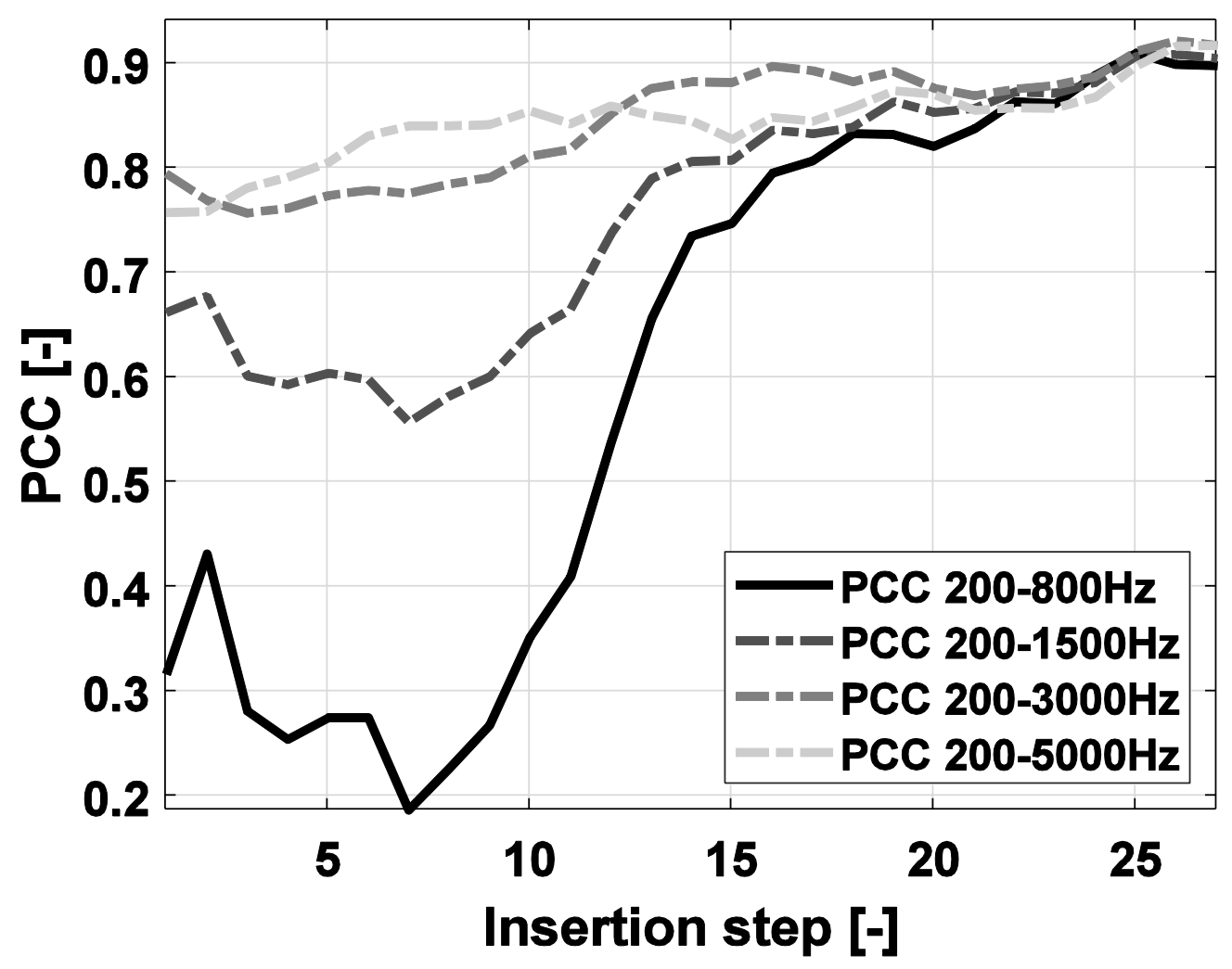

This article is protected by copyright. All rights reserved. 